\title{
Brain Gray Matter Decrease in Chronic Pain Is the Consequence and Not the Cause of Pain
}

\author{
Rea Rodriguez-Raecke, ${ }^{1}$ Andreas Niemeier, ${ }^{2}$ Kristin Ihle, ${ }^{1}$ Wolfgang Ruether, ${ }^{1}$ and Arne May ${ }^{1}$ \\ Departments of ${ }^{1}$ Systems Neuroscience and ${ }^{2}$ Orthopaedics, University Medical Center Hamburg Eppendorf, D-20246 Hamburg, Germany
}

Recently, local morphologic alterations of the brain in areas ascribable to the transmission of pain were reported in patients suffering from chronic pain. Although some authors discussed these findings as damage or loss of brain gray matter, one of the key questions is whether these structural alterations in the cerebral pain-transmitting network precede or succeed the chronicity of pain. We investigated 32 patients with chronic pain due to primary hip osteoarthritis and found a characteristic gray matter decrease in patients compared with controls in the anterior cingulate cortex (ACC), right insular cortex and operculum, dorsolateral prefrontal cortex (DLPFC), amygdala, and brainstem. We then investigated a subgroup of these patients $(n=10) 6$ weeks and 4 months after total hip replacement surgery, monitoring whole brain structure. After surgery, all 10 patients were completely pain free and we observed a gray matter increase in the DLPFC, ACC, amygdala, and brainstem. As gray matter decrease is at least partly reversible when pain is successfully treated, we suggest that the gray matter abnormalities found in chronic pain do not reflect brain damage but rather are a reversible consequence of chronic nociceptive transmission, which normalizes when the pain is adequately treated.

\section{Introduction}

Structural neuroimaging has begun to provide insights into the pathophysiology of pain syndromes. Several independent studies have suggested a decrease in gray matter in pain-transmitting areas in chronic pain patients (May, 2008). Altered brain morphology in areas related to pain processing are described in patients with phantom pain (Draganski et al., 2006), chronic back pain (Apkarian et al., 2004; Schmidt-Wilcke et al., 2006), fibromyalgia (Kuchinad et al., 2007; Schmidt-Wilcke et al., 2007; Hsu et al., 2009), complex regional pain syndrome (Geha et al., 2008), irritable bowl syndrome (Davis et al., 2008), tension-type headache, and migraine (Schmidt-Wilcke et al., 2005, 2008; Kim et al., 2008; Valfrè et al., 2008). Some authors discuss these data as atrophy, reinforcing the idea of damage or loss of brain gray matter (Apkarian et al., 2004; Rocca et al., 2006; Kuchinad et al., 2007; Kim et al., 2008). This interpretation is supported by the fact that most studies found a more or less significant correlation between brain gray matter changes and duration of pain (Apkarian et al., 2009). However, the exact process underlying these structural changes remains obscure and cell atrophy or synaptic loss as well as simple decreases in cell size or blood volume has also been suggested as possible explanations (Draganski and May, 2008; May, 2008). Independent of the exact nature of these changes, it is now generally accepted that chronic pain patients have a decrease

Received July 30, 2009; revised Sept. 8, 2009; accepted Sept. 10, 2009.

This work was supported by grants from the Deutsche Forschungsgemeinschaft (MA 1862/2-3) and Bundesministerium für Bildung und Forschung (37157 01 and Neurolmage Nord). We thank all volunteers for the participation in this study and the Physics and Methods group at Neurolmage Nord in Hamburg.

Correspondence should be addressed to Dr. Arne May, Assistant Professor of Neurology, Department of Systems Neuroscience, University of Hamburg Eppendorf, Martinistrasse 52, D-20246 Hamburg, Germany. E-mail: a.may@uke.uni-hamburg.de.

DOI:10.1523/JNEUROSCI.3687-09.2009

Copyright $\odot 2009$ Society for Neuroscience $\quad$ 0270-6474/09/2913746-05\$15.00/0 in gray matter in common, and while the exact loci differ between groups, there seems to be overlap in the cingulate cortex, insula, and dorsolateral prefrontal cortex (DLPFC) (Apkarian et al., 2004; Schmidt-Wilcke et al., 2005, 2006; Draganski et al., 2006; Kuchinad et al., 2007). These regions function as multi-integrative structures during the experience and the anticipation of pain. Considering that pain is a universal experience, it is not understood why only a relatively small proportion of humans develop a chronic pain syndrome. The question arises whether in some humans a structural difference in central pain-transmitting systems may act as a diathesis for chronic pain (Teutsch et al., 2008; May, 2009a). In the course of chronification, numerous modulatory mechanisms, such as effects at the nociceptor level, sympathetically maintained pain, the "wind-up" phenomenon, central sensitization, and changes in descending and ascending central modulatory mechanisms for the perception of pain have been postulated and altogether addressed as "neuronal plasticity" (Woolf and Salter, 2000). Some recent data suggest that structural changes of the brain may be added to this list (May, 2009b). There are no conclusive data regarding the cause of the different cortical and subcortical morphological changes that have been observed in chronic pain states, although the appearance of gray matter changes in phantom pain due to amputation (Draganski et al., 2006) and spinal cord injury (Wrigley et al., 2009) strongly suggests that the morphological changes are, at least in part, secondary to constant pain. Unfortunately, all available studies compared cohorts of patients and therefore no statement can be made with regard to dynamic changes.

Focusing on this aspect, we evaluated changes in brain structure using high-field magnetic resonance imaging (MRI) in 32 patients with unilateral primary hip osteoarthritis (OA) and severe permanent hip pain that were scheduled for total hip replacement (THR) surgery. The pain in hip OA is the only chronic 
Table 1. Demographic details of 10 patients with primary hip osteoarthritis undergoing total hip replacement surgery

\begin{tabular}{|c|c|c|c|c|c|c|c|c|c|}
\hline V & Sex & $\begin{array}{l}\text { Age } \\
\text { (years) }\end{array}$ & $\begin{array}{l}\text { Pain score } \\
\text { before surgery }\end{array}$ & $\begin{array}{l}\text { Pain duration } \\
\text { (years) }\end{array}$ & $\begin{array}{l}\text { Side of } \\
\text { pain }\end{array}$ & $\begin{array}{l}\text { Pain medication } \\
\text { before surgery }\end{array}$ & $\begin{array}{l}\text { Type of } \\
\text { medication }\end{array}$ & $\begin{array}{l}\text { Pain score } \\
\text { after surgery }\end{array}$ & $\begin{array}{l}\text { Pain medication } \\
\text { after surgery }\end{array}$ \\
\hline 1 & M & 66 & 80 & 4 & $\mathrm{R}$ & Yes & Diclofenac & 0 & No \\
\hline 2 & $\mathrm{~F}$ & 74 & 90 & 1 & $\mathrm{R}$ & No & n.a. & 0 & No \\
\hline 3 & $\mathrm{~F}$ & 66 & 80 & 4 & $\mathrm{R}$ & Yes & Diclofenac & 0 & No \\
\hline 4 & $\mathrm{~F}$ & 60 & 70 & 18 & $\mathrm{R}$ & Yes & Ibuprofen & 0 & No \\
\hline 5 & M & 66 & 60 & 1 & $\mathrm{~L}$ & Yes & Diclofenac & 0 & No \\
\hline 6 & M & 49 & 90 & 8 & L & Yes & Acemetacin, Sulfasalacin & 0 & No \\
\hline 7 & M & 80 & 50 & 3 & $\mathrm{~L}$ & No & n.a & 0 & No \\
\hline 8 & $\mathrm{~F}$ & 72 & 75 & 1 & $\mathrm{~L}$ & Yes & Ibuprofen, Verapamil & 0 & No \\
\hline 9 & $\mathrm{~F}$ & 44 & 60 & 3 & $\mathrm{~L}$ & No & n.a & 0 & No \\
\hline 10 & $F$ & 69 & 40 & 5 & $\mathrm{R}$ & Yes & Diclofenac & 0 & No \\
\hline
\end{tabular}

Pain score is given as values of a numerical rating scale anchored at $0=$ no pain and $100=$ worst pain ever. $M$, Male; $F$, female; $R$, right; $L$, left; n.a., not applicable.

Table 2. Significant changes in gray matter density in patients with chronic pain due to primary hip osteoarthritis

\begin{tabular}{|c|c|c|c|c|c|c|c|c|c|c|c|c|}
\hline \multirow[b]{2}{*}{ Anatomical location } & \multicolumn{3}{|c|}{$\begin{array}{l}\text { Patients }<\text { controls } \\
\text { MNI coordinates }\end{array}$} & \multirow[b]{2}{*}{$p$} & \multirow[b]{2}{*}{ Peak $t$ score } & \multirow[b]{2}{*}{ Number of voxels } & \multicolumn{3}{|c|}{$\begin{array}{l}\text { Testing periods } 1,2<3 \\
\text { MNI coordinates }\end{array}$} & \multirow[b]{2}{*}{$p$} & \multirow[b]{2}{*}{ Peak $t$ score } & \multirow[b]{2}{*}{ Number of voxels } \\
\hline & $x$ & $y$ & $Z$ & & & & $x$ & $y$ & $Z$ & & & \\
\hline L anterior cingulate cortex & -4 & 39 & 3 & 0.000 & 4.48 & 3401 & -2 & 34 & 0 & 0.007 & 2.75 & 813 \\
\hline R amygdala & 28 & 1 & -15 & 0.001 & 3.31 & 4 & 28 & 0 & -13 & 0.001 & 3.83 & 11,161 \\
\hline R DLPFC & 23 & 49 & 38 & 0.000 & 3.79 & 226 & 23 & 31 & 40 & 0.008 & 2.64 & 877 \\
\hline L midcingulate cortex & -11 & -14 & 35 & 0.000 & 4.02 & 75 & -17 & -18 & 50 & 0.007 & 2.75 & 219 \\
\hline R insular cortex & 51 & 17 & 1 & 0.000 & 5.38 & 4468 & 36 & 9 & 1 & 0.004 & 2.99 & 11,161 \\
\hline L insular cortex & -41 & 26 & 4 & 0.000 & 4.02 & 287 & -44 & 27 & -13 & 0.010 & 2.53 & 712 \\
\hline Brainstem & 2 & -30 & -48 & 0.000 & 3.72 & 20 & 0 & -28 & -42 & 0.008 & 2.68 & 1063 \\
\hline L medial temporal gyrus/S2 & -62 & -17 & 0 & 0.000 & 4.07 & 1201 & -58 & -5 & -10 & 0.012 & 2.47 & 493 \\
\hline$R$ anterior cingulate cortex & 12 & 23 & 24 & 0.000 & 4.19 & 326 & 2 & 33 & 1 & 0.009 & 2.39 & 813 \\
\hline L midorbital gyrus & -9 & 24 & -22 & 0.000 & 4.65 & 1248 & & & & & & \\
\hline R midorbital gyrus & 6 & 62 & -12 & 0.000 & 5.10 & 776 & & & & & & \\
\hline R superior frontal gyrus/area 6 & 21 & -6 & 59 & 0.000 & 4.13 & 166 & & & & & & \\
\hline R medial temporal pole & 27 & 9 & -42 & 0.000 & 3.69 & 49 & & & & & & \\
\hline R cerebellum & 18 & -43 & -33 & 0.000 & 4.64 & 1544 & & & & & & \\
\hline R superior medial gyrus & 9 & 66 & 13 & 0.000 & 4.17 & 244 & & & & & & \\
\hline R area 2/S1 & 54 & -32 & 53 & 0.000 & 3.92 & 161 & & & & & & \\
\hline
\end{tabular}

The changes are tabulated in terms of the brain region and the corresponding Brodmann's area. The $x, y, z$ coordinates are according to the Montreal Neurological Institute (MNI) atlas. Each location is the peak within a cluster (defined as the voxel with the highest $z$ score). The left side of the table ("patients $<$ controls") lists significant decreases of gray matter between patients and controls with a statistical threshold set at $p<0.001$ uncorrected. The right side of the table ("testing periods $1,2<3^{\prime \prime}$ ) lists corresponding areas showing a gray matter increase in 10 pain-free patients at the third scanning period $12-18$ weeks after total hip replacement surgery, as compared to the first (preoperative) and second (6 weeks post surgery) scans ( $p<0.05$ uncorrected). R, Right side; L, left side.

pain syndrome which is principally curable, as $88 \%$ of these patients are regularly free of pain following THR (Nikolajsen et al., 2006). We therefore investigated a subgroup of these patients $(n=10)$ after successful THR and monitored structural brain changes after $\sim 6$ weeks and 4 months, focusing on possible increases of gray matter over time.

\section{Materials and Methods}

\section{Volunteers}

Thirty-two patients with unilateral primary hip OA (18 with pain on the right side, 14 with pain on the left side) were recruited (mean age $66.8 \pm$ 9.0 years, 19 female) and compared with an age- and gender-matched healthy control group (mean age 63.9 \pm 8.8 years, 19 female). All patients with primary hip OA had a pain history of longer than 12 months, ranging from 1 to 33 years (mean 7.35 years). We also assessed any occurrence of minor pain events, including toothache, earache, and headache up to 4 weeks before the study. None of the patients or volunteers had any neurological or internal medical history, and three patients showed mild to moderate depressive symptoms according to Beck's Depression Inventory (BDI), but none qualified for major depression or other psychiatric diseases according to the Diagnostic and Statistic Manual of Mental Disorder IV. None of the controls reported any acute or chronic pain and none showed any depressive symptoms according to BDI. A subgroup of 10 patients ( $n=10$, mean age 64.6 years, mean duration of pain 4.8 years; for demographic details, see Table 1) was again investigated 6 and 16 weeks after THR, when the patients in this subgroup were completely pain free. The study was given approval by the local ethics committee and written informed consent was obtained from all study participants before examination.

Voxel-based morphometry: data acquisition

Image acquisition. High-resolution MR scanning was performed on a $3 \mathrm{~T}$ MRI system (Siemens Trio) with a standard head coil. For each time point, day 1 (before surgery), as well as 6-8 weeks and 16-18 weeks after surgery, a $\mathrm{T}_{1}$ weighted structural MRI was acquired for each patient by using a 3D-FLASH sequence (TR $15 \mathrm{~ms}$, TE $4.9 \mathrm{~ms}$, flip angle $25^{\circ}, 1 \mathrm{~mm}$ slices, FOV $256 \times 256$; 240 slices).

Image processing and statistical analysis. Data preprocessing and analysis were performed with SPM2 (Welcome Department of Cognitive Neurology, London, UK) running under Matlab (Mathworks) and containing a voxel-based morphometry (VBM) toolbox for longitudinal data (http://dbm.neuro.uni-jena.de/) that is based on high-resolution structural 3D MR images and allows for applying voxelwise statistics to detect regional differences in gray matter volumes (Ashburner and Friston, 2000; Good et al., 2001). In summary, preprocessing involved spatial normalization, gray matter segmentation, and $10 \mathrm{~mm}$ spatial smoothing with a Gaussian kernel. For the preprocessing steps, we used an optimized protocol (Ashburner and Friston, 2000; Good et al., 2001) and a scanner- and study-specific gray matter template. A voxel-by-voxel repeated-measures ANOVA was used to detect regional differences in gray matter between cohorts and over all three scans in the operated patient group. We tested for any regions that showed an increase or decrease in brain structure between groups [patients $(n=32)$ and con- 
trols $(n=32)]$ and in the longitudinal group ( $n=10)$ between the first two scans (before the operation and 6-8 weeks after the operation) with the third scan (pain free 16-18 weeks after the operation). We applied a threshold of $p<$ 0.001 (uncorrected) across the whole brain. The groups were closely matched for age and sex with no significant differences between the groups, and, therefore, no age or sex confounds were included. To address possible differences between patients with right- and left-sided pain, we flipped the datasets of patients with left sided osteoarthritis $(n=13)$. In the cohort analysis but not in the longitudinal analysis we performed a correction for volume changes (modulation) by modulating each voxel with the Jacobian determinants derived from the spatial normalization, allowing us to also test for regional differences in the absolute amount of gray matter. Because any brain changes due to chronic pain will need some time to recede following operation and cessation of pain, we compared in the longitudinal analysis scans 1 and 2 with scan 3 . Only for regions which showed a significant gray matter decrease in the patient group compared with the control group (i.e., the DLPFC, ACC, amygdala, insula, and brainstem), we accepted an uncorrected threshold of 0.05 across the whole brain.

\section{Results}

Patients with primary hip OA $(n=32)$ showed preoperatively reduced gray matter density in anterior cingulate cortex (ACC), the orbitofrontal cortex, right insular cortex and operculum, right midor-

bital gyrus, left superior medial gyrus, and brainstem compared with age- and gender-matched controls (Table 2, Fig. 1). Except for the left middle frontal gyrus $(x=-28 ; y=9 ; z=62 ; t=4.07 ; p<$ 0.001 uncorrected), no significant increase in gray matter density was found in patients with OA. Flipping the datasets of the patients with left-sided osteoarthritis and therefore normalizing for the side of the pain did not change the results, but improved the significance for some of the areas (such as the right insular cortex). We did not find any differences between modulated and nonmodulated data. In the longitudinal analysis, i.e., comparing the first scan (preoperative) to the second scan (postoperative), there was no significant increase in gray matter volume, probably due to the relatively short time period after surgery. A significant increase of gray matter was detected by comparing the first and second scans (chronic pain) with the third scan (pain free) in the ACC, DLPFC, amygdala, brainstem, and right insular cortex in the patients with OA (Fig. 2).

\section{Discussion}

Using VBM we found significant differences in CNS gray matter in chronic pain patients due to primary hip osteoarthritis (Fig. 1), which fall into two broad groups: (1) areas showing a decrease in gray matter comparing the chronic pain state in patients and pain-free controls mainly involving the ACC, DLPFC, amygdala, insular cortex, orbital gyrus, and brainstem; and (2) areas showing an increase in gray matter over time after pain relief in a subgroup of these patients, such as the ACC, DLPFC, amygdala, brainstem, and insular cortex.

These data suggest that prolonged nociceptive input due to primary hip osteoarthritis leads to an alteration in morphology and/or cytoarchitecture of anterograde projection areas, which partly recede when the patient is pain free after endoprosthetic joint replacement surgery. At least in these patients, it is unlikely that repeated nociceptive input leading to chronicity of pain over time results in irreversible "damage" and/or "atrophy" to the brain.

In line with the literature, we found no changes in M1 or SMA between patients and controls in our cohort study. Of note, nearly all authors who investigated pain and brain morphometry noticed changes in pain-transmitting areas but not in motor areas, although chronic pain often hinders physical exercise. Perhaps this is the reason why we did not see any increase in these areas over time because they were not initially altered. One could reason that the qualitative change (i.e., pain or no pain) is more critical for the brain to change its structure than a quantitative change of day-to-day activity (e.g., more or less exercise). Interestingly, neither performance nor exercise of a visio-motor task was able to predict structural changes in the brain (Driemeyer et al., 2008). Recently, it was shown that cognitive behavioral therapy in patients with fatigue syndrome increases gray matter volume in the lateral prefrontal cortex. This structural change was positively correlated to improvements in cognitive speed in these patients (de Lange et al., 2008).

Regarding chronic pain, a lot of thought has been devoted to considering changes in primary afferents, dorsal root ganglia, and spinal cord dorsal horn (Woolf and Salter, 2000). Very recent data added functional (Flor et al., 2006) and structural (May, 2008; Apkarian et al., 2009) changes of the brain to the list of alterations. A striking feature of recently published structural brain changes in chronic pain syndromes is the fact that the gray matter changes were not randomly distributed but concerned 

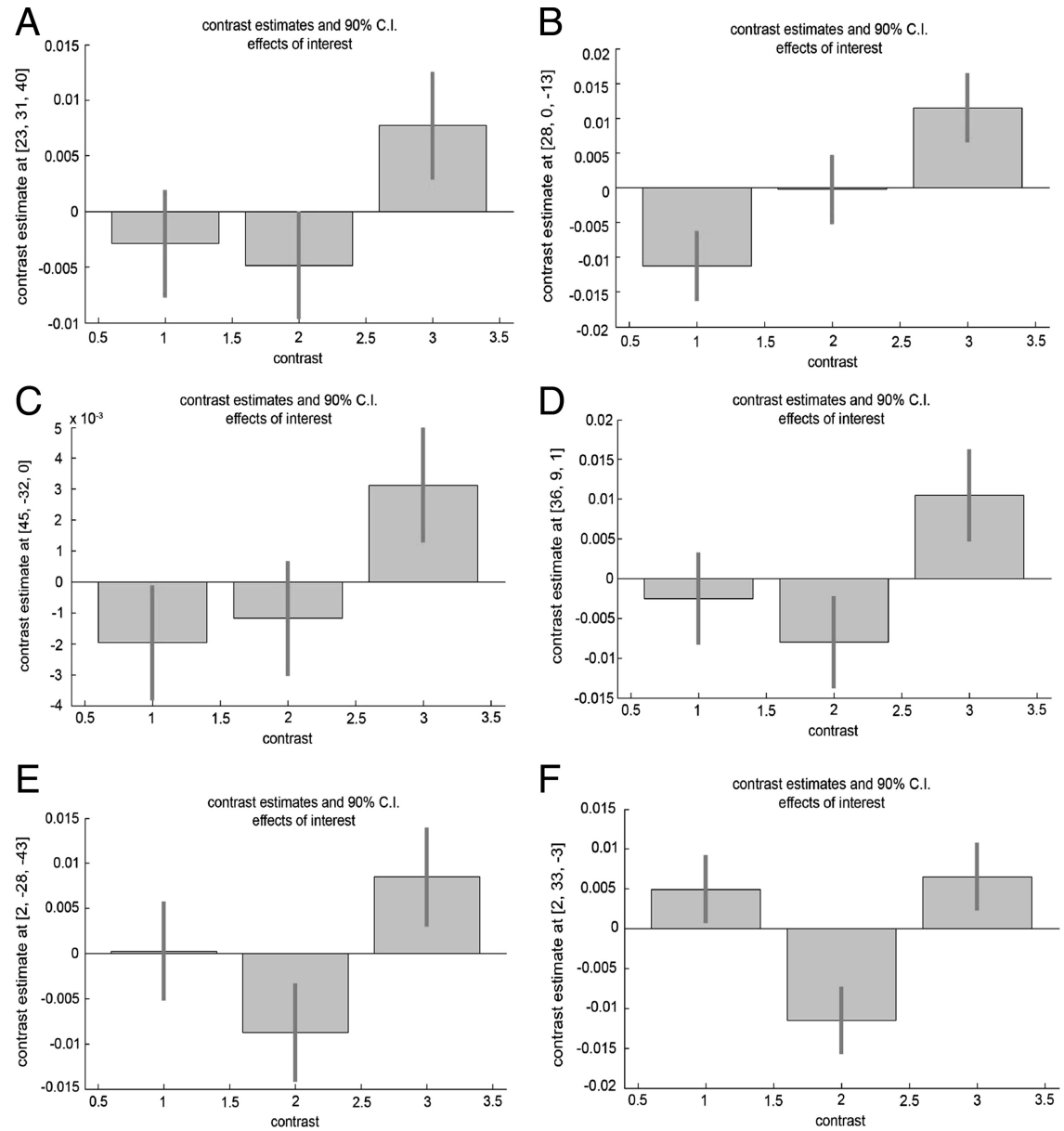

Figure 2. Gray matter changes in the longitudinal group $(n=10)$ between the first two scans (before the operation and $6-8$ weeks after the operation) with the third scan (pain free 16-18 weeks after the operation). Each box plot represents relative gray matter percentage signal change over time and $90 \%$ confidence interval (gray line) averaged over the cluster of the dorsolateral prefrontal cortex $(\boldsymbol{A})$, amygdala $(\boldsymbol{B})$, S2 cortex $(\boldsymbol{C})$, insular cortex $(\boldsymbol{D})$, brainstem $(\boldsymbol{E})$, and anterior cingulate cortex $(\boldsymbol{F})$. C.I., Confidence interval.

defined and functionally highly specific brain areas, namely, involvement in supraspinal nociceptive processing. The most prominent findings were different for each pain syndrome but overlapped in the cingulate cortex, the DLPFC, the insula, and dorsal pons (May, 2008), regions which we also find to be involved in chronic pain due to hip OA. The central question is whether a population difference between patients and controls arises as a consequence of chronic pain, contributes to the neurobiological basis of the chronification of pain, or both. This question is not redundant, as a degenerative process is irreversible.

In vivo demonstrations of a change in brain structure could represent a neuroanatomical substrate for the respective disease (Reiss et al., 2004) or just an epiphenomenon or even an artifact. Given that changes in the periphery, i.e., loss of afferent input due to unilateral amputation of an extremity (Draganski et al., 2006) or phantom pain due to spinal cord injury (Wrigley et al., 2009) and even amblyopia (Mendola et al., 2005) and strabismus (Chan et al., 2004) may change the brain structure of individuals, one has to conclude that these changes are possibly a result of experience-dependent neuronal plasticity (Draganski and May, 2008). Our data provide evidence for activation-dependant brain plasticity in humans on a structural level (Draganski et al., 2004; Boyke et al., 2008) as the key to structural changes of the brain in chronic pain patients. Using voxel-based morphometry, it was demonstrated that repeated painful stimulation resulted in a substantial increase of gray matter in classical somatosensory areas, including midcingulate and somatosensory cortex (Teutsch et al., 2008). This finding follows the previously described functional pattern (Bingel et al., 2008) precisely, i.e., a significant change during the protocol which reverses to prestimulation levels after 1 year. It is an intriguing fact that chronic pain patients suffer constant pain but seem not to develop an increase in gray matter in somatosensory areas, although several studies have shown that exercise is accompanied by an increase of gray matter in the regions which are specific for the respective task (for review, see May, 2009b). One explanation for this lack of gray matter increase in somatosensory areas in chronic pain patients is that they do not have a significant noxious input (any more). In that case, the experience of constant pain is mostly driven by the brain itself and the afferent (peripheral noxious) input is no longer needed for this experience. However, the present study suggests that the main difference in the brain structure between pain patients and controls may recede when the pain is cured. Although we have controlled for affect and depression scores, the question arises of how many other behavioral changes due to the absence of pain, such as enhancement of social contacts, agility, physical training, and uncountable other lifestyle changes may contribute to the results (decrease of gray matter in chronic pain as well as increase of gray matter when pain is gone).

Another factor which may bias our interpretation of the results is the fact that nearly all patients took medication against pain, which they stopped when they were pain free following successful surgery and rehabilitation. One could argue that nonsteroidal anti-inflammatory drugs (NSAIDs) such as diclofenac or ibuprofen have some effects on neural systems (Vanegas and Tortorici, 2002) and that the impact of pain killers on morphometric findings should not be underestimated. However, no study so far has shown effects of NSAIDs on brain morphology, but several papers found that changes in brain structure in chronic pain patients are solely explained neither by pain-related inactivity (Draganski et al., 2006) nor by pain medication (Apkarian et al., 2004; Schmidt-Wilcke et al., 2005; Kuchinad et al., 2007). However, specific studies are lacking and pain medication may well have a specific impact on our findings.

Our data reflect real life, and it is not possible to delineate which of the above-mentioned factors contributes to the genesis of the change in brain structure. In our patients we found no meaningful changes in the interim scan after the operation, although one would expect changes following the physiotherapy regimen after the operation. Further studies with more patients and using perhaps an even longer follow-up frame are certainly warranted. Nevertheless, central to our findings is that changes in nociceptive input and transmission lead to intracortical remod- 
elling and that these changes are not irreversible. These data highlight the remarkable potential of the adult brain to undergo anatomical changes that have a great impact on its functioning. Improved understanding of experience-dependent changes in cortical plasticity may have vast clinical implications for the treatment of chronic pain, focusing on use-dependent plasticity to improve mobility, alleviating pain, and increasing quality of life.

\section{References}

Apkarian AV, Sosa Y, Sonty S, Levy RM, Harden RN, Parrish TB, Gitelman DR (2004) Chronic back pain is associated with decreased prefrontal and thalamic gray matter density. J Neurosci 24:10410-10415.

Apkarian AV, Baliki MN, Geha PY (2009) Towards a theory of chronic pain. Prog Neurobiol 87:81-97.

Ashburner J, Friston KJ (2000) Voxel-based morphometry-the methods. Neuroimage 11:805-821.

Bingel U, Herken W, Teutsch S, May A (2008) Habituation to painful stimulation involves the antinociceptive system-a 1-year follow-up of 10 participants. Pain 140:393-394.

Boyke J, Driemeyer J, Gaser C, Büchel C, May A (2008) Training-induced brain structure changes in the elderly. J Neurosci 28:7031-7035.

Chan ST, Tang KW, Lam KC, Chan LK, Mendola JD, Kwong KK (2004) Neuroanatomy of adult strabismus: a voxel-based morphometric analysis of magnetic resonance structural scans. Neuroimage 22:986-994.

Davis KD, Pope G, Chen J, Kwan CL, Crawley AP, Diamant NE (2008) Cortical thinning in IBS: implications for homeostatic, attention, and pain processing. Neurology 70:153-154.

de Lange FP, Koers A, Kalkman JS, Bleijenberg G, Hagoort P, van der Meer JW, Toni I (2008) Increase in prefrontal cortical volume following cognitive behavioural therapy in patients with chronic fatigue syndrome. Brain 131:2172-2180.

Draganski B, May A (2008) Training-induced structural changes in the adult human brain. Behav Brain Res 192:137-142.

Draganski B, Gaser C, Busch V, Schuierer G, Bogdahn U, May A (2004) Neuroplasticity: changes in grey matter induced by training. Nature 427:311-312.

Draganski B, Moser T, Lummel N, Gänssbauer S, Bogdahn U, Haas F, May A (2006) Decrease of thalamic gray matter following limb amputation. Neuroimage 31:951-957.

Driemeyer J, Boyke J, Gaser C, Büchel C, May A (2008) Changes in gray matter induced by learning-revisited. PLoS One 3:e2669.

Flor H, Nikolajsen L, Staehelin Jensen T (2006) Phantom limb pain: a case of maladaptive CNS plasticity? Nat Rev Neurosci 7:873-881.

Geha PY, Baliki MN, Harden RN, Bauer WR, Parrish TB, Apkarian AV (2008) The brain in chronic CRPS pain: abnormal gray-white matter interactions in emotional and autonomic regions. Neuron 60:570-581.

Good CD, Johnsrude IS, Ashburner J, Henson RN, Friston KJ, Frackowiak RS (2001) A voxel-based morphometric study of ageing in 465 normal adult human brains. Neuroimage 14:21-36.

Hsu MC, Harris RE, Sundgren PC, Welsh RC, Fernandes CR, Clauw DJ, Williams DA (2009) No consistent difference in gray matter volume between individuals with fibromyalgia and age-matched healthy subjects when controlling for affective disorder. Pain 143:262-267.
Kim JH, Suh SI, Seol HY, Oh K, Seo WK, Yu SW, Park KW, Koh SB (2008) Regional grey matter changes in patients with migraine: a voxel-based morphometry study. Cephalalgia 28:598-604.

Kuchinad A, Schweinhardt P, Seminowicz DA, Wood PB, Chizh BA, Bushnell MC (2007) Accelerated brain gray matter loss in fibromyalgia patients: premature aging of the brain? J Neurosci 27:4004-4007.

May A (2008) Chronic pain may change the structure of the brain. Pain 137:7-15.

May A (2009a) New insights into headache: an update on functional and structural imaging findings. Nat Rev Neurol 5:199-209.

May A (2009b) Morphing voxels: the hype around structural imaging of headache patients. Brain 132:1419-1425.

Mendola JD, Conner IP, Roy A, Chan ST, Schwartz TL, Odom JV, Kwong KK (2005) Voxel-based analysis of MRI detects abnormal visual cortex in children and adults with amblyopia. Hum Brain Mapp 25:222-236.

Nikolajsen L, Brandsborg B, Lucht U, Jensen TS, Kehlet H (2006) Chronic pain following total hip arthroplasty: a nationwide questionnaire study. Acta Anaesthesiol Scand 50:495-500.

Reiss AL, Eckert MA, Rose FE, Karchemskiy A, Kesler S, Chang M, Reynolds MF, Kwon H, Galaburda A (2004) An experiment of nature: brain anatomy parallels cognition and behavior in Williams syndrome. J Neurosci 24:5009-5015.

Rocca MA, Ceccarelli A, Falini A, Colombo B, Tortorella P, Bernasconi L, Comi G, Scotti G, Filippi M (2006) Brain gray matter changes in migraine patients with T2-visible lesions: a 3-T MRI study. Stroke 37:1765-1770.

Schmidt-Wilcke T, Leinisch E, Straube A, Kämpfe N, Draganski B, Diener HC, Bogdahn U, May A (2005) Gray matter decrease in patients with chronic tension type headache. Neurology 65:1483-1486.

Schmidt-Wilcke T, Leinisch E, Gänssbauer S, Draganski B, Bogdahn U, Altmeppen J, May A (2006) Affective components and intensity of pain correlate with structural differences in gray matter in chronic back pain patients. Pain 125:89-97.

Schmidt-Wilcke T, Luerding R, Weigand T, Jurgens T, Schuierer G, Leinisch E, Bogdahn U (2007) Striatal grey matter increase in patients suffering from fibromyalgia-a voxel-based morphometry study. Pain 132 [Suppl 1]:S109-S116.

Schmidt-Wilcke T, Ganssbauer S, Neuner T, Bogdahn U, May A (2008) Subtle grey matter changes between migraine patients and healthy controls. Cephalalgia 28:1-4.

Teutsch S, Herken W, Bingel U, Schoell E, May A (2008) Changes in brain gray matter due to repetitive painful stimulation. Neuroimage $42: 845-849$

Valfrè W, Rainero I, Bergui M, Pinessi L (2008) Voxel-based morphometry reveals gray matter abnormalities in migraine. Headache 48:109-117.

Vanegas H, Tortorici V (2002) Opioidergic effects of nonopioid analgesics on the central nervous system. Cell Mol Neurobiol 22:655-661.

Woolf CJ, Salter MW (2000) Neuronal plasticity: increasing the gain in pain. Science 288:1765-1769.

Wrigley PJ, Gustin SM, Macey PM, Nash PG, Gandevia SC, Macefield VG, Siddall PJ, Henderson LA (2009) Anatomical changes in human motor cortex and motor pathways following complete thoracic spinal cord injury. Cereb Cortex 19:224-232. 